Case Report

\title{
NON-FORMATION OF THE MAIN TRUNK OF THE SCIATIC NERVE AND UNUSUAL RELATIONSHIPS TO THE PIRIFORMIS MUSCLE
}

\author{
J. Stoyanov, N. Tomov, A. Georgieva, N. Dimitrov, L. Surchev, D. Sivrev* \\ Department of Anatomy, Faculty of Medicine, Trakia University, Stara Zagora, Bulgaria
}

\begin{abstract}
Background: The sciatic nerve is the largest branch of the sacral plexus. Variations of its origin, exit from the pelvis, emergence and branching in the posterior region of the thigh, especially in regards to the piriformis muscle, are an object of interest due to the possibility to be involved in the pathogenensis of clinically significant non-discogenic sciatica or piriformis syndrome.

Case report: We present a case of variant anatomy of the sciatic nerve, discovered during routine dissection of the left gluteal region of an adult female cadaver. We observed a non-formation of the main trunk of the nerve; rather, the tibial nerve passed inferiorly to the piriformis muscle, while the common peroneal nerve went through the body of the bifid piriformis muscle, immediately next to its tendon. The two branches continued their course in the thigh without joining and forming a proper sciatic nerve. The medical records of the body donor did not reveal any neurological impairment which could be linked to this anatomical peculiarity. Conclusion: The anatomy of the sciatic nerve could be considered to be a factor of clinical significance. The high prevalence of similar anatomical variations should be kept in mind during the diagnostic process of clinical entities involving the sciatic nerve.
\end{abstract}

Key words: Sciatic nerve, piriformis muscle, variation

\section{INTRODUCTION}

The sciatic nerve is the largest nerve in the human body, emerging from the sacral plexus and including nerve fibers from the ventral rami of spinal nerves L4 to S3. In most cases, the main trunk of the sciatic nerve forms just anteriorly to the piriformis muscle, then leaves the pelvis through the infrapiriform foramen and continues in the posterior region of the thigh, where it splits in its two main components - the tibial and the common peroneal nerves (1). As it courses from the pelvis to the posterior femoral region, it comes in close contact with the pelvitrochanteric muscle group, especially with the piriformis muscle. The close relationship of this muscle and the sciatic nerve could be the cause of nerve compression and the so called piriformis syndrome $(2,3)$. Nerve entrapment could be caused not only by irritation, inflammation or hypertrophy of the piriformis muscle, but also by aberrant anatomy of the sciatic nerve and its course between muscles $(4,5)$. Data from many case reports suggest this neuromuscular conflict to be at least a contributing factor of

\footnotetext{
*Correspondence to: $D$. Sivrev, Department of Anatomy, Faculty of Medicine, Trakia University, Stara Zagora, Bulgaria
}

the pathogenesis of very low back and buttock pain (6). Another practical consideration involving the anatomy of the sciatic nerve is the level of its division into tibial and common peroneal nerves. A high division of the nerve could potentially lead to incomplete nerve block when attempting popliteal block anesthesia (7).

\section{MATERIALS AND METHODS}

We dissected the left lower limb of a formalin fixed adult female cadaver, age 58, available at the Department of Anatomy, Faculty of Medicine, Trakia University, Stara Zagora, Bulgaria. The gluteal region was carefully cleaned, and after transection of the gluteus maximus muscle, the greater sciatic foramen, and the pelvitrochanteric group of muscles were exposed. The piriformis muscle, the supra- and infrapiriform foramens were demonstrated, and the nerves and vessels passing through the region were carefully followed and prepared.

\section{RESULTS}

During our dissection, we observed the piriformis muscle originating from the middle of the greater sciatic notch, and attaching with a single tendon to the greater trochanter. The 
tibial nerve was visualized emerging below the lower margin of the muscular part of the piriformis muscle, through the infrapiriform foramen. The common peroneal nerve, on the other hand, left the pelvic cavity through the bifid piriformis muscle, piercing its muscular body close to the musculotendinous junction (Figure 1).
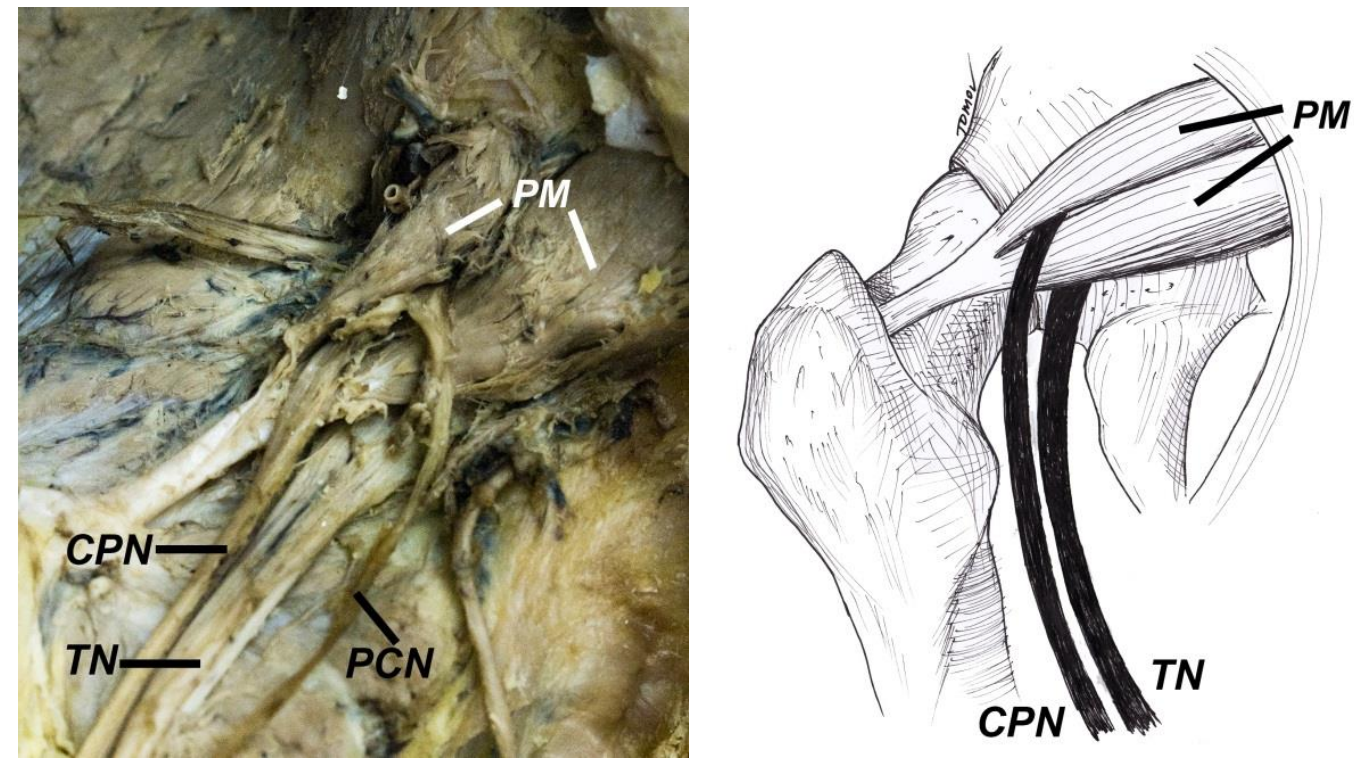

Figure 1. (A) Photograph and (B) schematic representation of the relationship of the piriformis muscle and the tibial and common peroneal nerves. $\mathrm{PM}$ - piriformis muscle; $\mathrm{TN}$ - tibial nerve; $\mathrm{CPN}$ - common peroneal nerve; $\mathrm{PCN}$ - posterior cutaneous nerve (of the thigh).

The two nerves continued their course distally, in the posterior region of the thigh, running in the groove between the biceps femoris and the semimembranosus and semitendinosus muscles, reaching the popliteal fossa. Their further path and branching did not differ from the common pattern. The piriformis muscle itself could be described as having two discrete heads - a smaller superior and a larger inferior, separated by a thin gap, through which the common peroneal nerve passed. The two heads of the muscle continued laterally to form a single round tendon attaching to the upper border of the greater trochanter of the femur, without associating with other muscular structures. Noteworthy is also the fact that the posterior femoral cutaneous nerve also appeared in the gap between the heads of the piriformis muscle as well, about $2 \mathrm{~cm}$ medially to the common peroneal nerve. The remaining topographic features of the gluteal region were unremarkable - the superior gluteal nerve and the homonymous vessels emerged above the bifid piriformis muscle, the pudendal nerve and the internal pudendal vessels passed through the infrapirifom foramen, just medially to the tibial nerve. During further dissection we did not observe any other anatomical variations in this cadaver. Furthermore, this morphological peculiarity could not be suggested to have any functional influence due to the lack of any signs of detriment, e.g. muscle atrophy or deformities.

\section{DISCUSSION}

Variations of the sciatic nerve and its branches in relation to the piriformis muscle are not uncommon. To our knowledge, this is the first recorded incidence of this variation observed during routine dissection at the Department of Anatomy in Stara Zagora, as well as the first report of a similar anatomical variation in a Bulgarian population. Other studies suggest variant anatomy of the sciatic nerve to be present in a highly variable percentage of cases (8).

Beaton and Anson have proposed a classification of the variations of the emergence of the sciatic nerve in relation to the piriformis muscle $(9,10)$ in six types, as follows:

Type 1: Undivided nerve below undivided muscle.

Type 2: Divisions of nerve through and below divided muscle.

Type 3: Divisions above and below undivided muscle.

Type 4: Undivided nerve between heads.

Type 5: Divisions between and above heads.

Type 6: Undivided nerve above undivided muscle 
The present case could be classified as a Type 2 variation according to the classification of Beaton and Anson, which reported and discussed the incidence of this variation in $11,7 \%$ of the extremities dissected. A recent study [11] reports a similar incidence of the same variation - 9\% of the sample. The relative portion of similar Type 2 variations is as low as $2,5 \%$ in a study conducted on fetuses (12). However, studies based on a Japanese population report the typical Type 1 anatomy to be present in only $60 \%$ of cases, while in the rest numerous variations were described, with Beaton and Anson Type 2 being the most common - 34\% of extremities (13). Populational differences in the pattern of sciatic nerve emergence from the pelvis have been noted as probable (14).

Furthermore, the position of the nerve relative to the tendon of the piriformis muscle may make it more or less prone to compression, e.g. in case of muscle inflammation - an aspect not elucidated by far. The position of the nerve branches inside the bifid muscle, relative to its tendon, are not addressed in the Beaton and Anson classification. Additionally, the presence of the posterior femoral cutaneous nerve in the space between the heads of the bifid piriformis muscle might contribute to thigh-referred pain caused by the neuromuscular conflict, which could be confused with sciatica or other pathology involving sensory phenomena along sacral dermatomes.

Plenty of case reports of patients with clinically significant aberrant anatomy and the observations of larger samples of symptomatic individuals exist. We lack data for functional impairment caused by the peculiarity observed. This could be interpreted as evidence towards hidden prevalence of anatomical variations among the general population. This neuromuscular relationship posesses individual features, such as the position of the nerves relative to the muscle, the size of the spaces they pass through, and the coincidence of variations of other nerves, e.g. the posterior femoral cutaneous nerve. They possibly play a functional role as well, adding more details to be considered then the included in the Beaton and Anson classification.

\section{REFERENCES}

1. Standring S (ed.) Chapter 110: General organization and surface anatomy of the lower limb. Chapter 111: Pelvic girdle, gluteal region and hip joint. In: Gray's
Anatomy, The anatomical basis of clinical practice. 39th ed: (pp. 1399-1459) Elsevier Churchill Livingstone, Edinburgh, 2005

2. Barton PM. Piriformis syndrome: A rational approach to management. Pain, 1991, 47:345-352.

3. Broadhurst NA, Simmons N, Bond MJ. Piriformis syndrome: Correlation of muscle morphology with symptoms and signs. Arch Phys Med Rehabil, 2004, 85:20362039 ,

4. Pecina M. Contribution to the etiological explanation of the piriformis syndrome. Acta Anat (Basel), 1979, 105:181-187.

5. Chen WS. Bipartite piriformis muscle: an unusual cause of sciatic nerve entrapment. Pain, 1994; 58:269-272.

6. Papadopoulos EC, Khan SN. Piriformis syndrome and low back pain: A new classification and review of the literature. Orthop Clin Am, 2004, 35:65-71.

7. Prakash, Bhardwaj AK, Devi MN, Sridevi NS, Rao PK, Singh G. Sciatic nerve division: a cadaver study in the Indian population and review of the literature. Singapore Med J; 2010, 51:721-723.

8. Pokorny D, Jahoda D, Veigl D, Pinskerova $\mathrm{V}$, Sonsa A. Topographic variations of the relationship of the sciatic nerve and the piriformis muscle and its relevance to palsy after total hip arthroplasty. Surg Radiol Anat, 2006, 28:88-91.

9. Beaton LE. The sciatic nerve and piriform muscle: their interrelations possible cause of coccgodynia. J Bone Joint Surg Am, 1938, 20:686-688.

10.Beaton LE, Anson BJ. The relation of the sciatic nerve and its subdivisions to the Piriformis muscle. Anat Rec, 1937, 70:1-5.

11.Berihu BA, Debeb YG. Anatomical variation in bifurcation and trifurcations of sciatic nerve and its clinical implications: in selected university in Ethiopia. BMC Res Notes, 2015, 8:633.

12.Ugrenovic S, Jovanovic I, Krstic V, Stojanovic V, Vasovic L, Antic S, Pavlovic $\mathrm{S}$. The level of the sciatic nerve division and its relations to the piriform muscle. Vojnosanit Pregl, 2005, 62:45-49.

13. Chiba S. Multiple positional relationships of nerves arising from the sacral plexus to the piriformis muscle in humans. Kaibogaku Zasshi, 1992, 67: 691-724.

14.Güvençer M, Iyem C, Akyer P, Tetik S, Naderi S. Variations in the high division of the sciatic nerve and relationship between the sciatic nerve and the piriformis. Turkish Neurosurg, 2009, 19:139-144. 